\title{
Variación espacio-temporal de diversidad y densidad de los poliquetos pelágicos en la Bahía de Acapulco, Guerrero, México
}

\author{
Fernández-Álamo M. A. ${ }^{1}$, Gálvez-Zeferino N. ${ }^{2}$, Roa-Venicio, M. ${ }^{1}$ \& Rojas-Herrera A. ${ }^{3}$ \\ 1. Laboratorio de Invertebrados, Facultad de Ciencias, UNAM; mafa@ciencias.unam.mx \\ 2. Centro Interdisciplinario de Ciencias Marinas, Instituto Politécnico Nacional, La Paz, Baja California Sur, México \\ 3. Facultad de Ecología Marina, Universidad Autónoma de Guerrero, Guerrero, México
}

Recibido 28-XI-2018. Corregido 22-V-2019. Aceptado 30-VI-2019.

\begin{abstract}
Spatio-temporal variation of the diversity and density of the pelagic polychaetes in Acapulco Bay, Guerrero, Mexico. Introduction: From a global point of view, polychaetes that inhabit in the pelagic community have been little studied, although they are an important link in the food chains of marine ecosystems. Objective: to analyze the local richness and density of polychaetes. Methods: Plankton samples were taken in 17 localities during May and December 2013, through surface trawls in a barge with an outboard motor. We used a net with $0.3 \mathrm{~m}$ mouth diameter, $1.30 \mathrm{~m}$ long and a $0.3 \mathrm{~mm}$ aperture mesh. From each collected sample, polychaetes were separated and determined at the lowest possible taxonomic level. Results: We collected a total of 1873 individuals, with an important variation in relative density, since in May $7952.0 \mathrm{ind} / 100 \mathrm{~m}^{3}$ were recorded, while in December there were only $882.1 \mathrm{ind} / 100 \mathrm{~m}^{3}$. The differences in the composition and density of the determined polychaetes, were analyzed, of which the highest proportion belongs to the larval forms of the benthic (meroplankton) populations, and the lowest proportion to holoplankton organisms. During the two periods of study were observed thirteen families, from which Sabellariidae, Spionidae, Chaetopteridae, Magelonidae, Iospilidae and Typhloscolecidae were common in both periods, while Aphroditidae, Syllidae, Phyllodocidae and Alciopidae were observed only in May, and Serpulidae, Sabellidae and Lopadorhynchidae were observed only in December. Conclusions: It is important to point out that the observed variations provide valuable information about the life cycles and the biogeographic aspects of the polychaetes in the study area.
\end{abstract}

Key words: marine worms, zooplankton, ecology, Mexican Pacific.

Fernández-Álamo M. A., Gálvez-Zeferino N., Roa-Venicio, M., \& Rojas-Herrera A. (2019). Variación espacio-temporal de diversidad y densidad de los poliquetos pelágicos en la Bahía de Acapulco, Guerrero, México. Revista de Biología Tropical, 67(S5) Suplemento, S92-S100.

A pesar de la importancia del zooplancton en los aspectos ecológicos fundamentales del ecosistema marino, como es su papel en las redes alimentarias oceánicas (depredadores y presas) y con ello su enorme contribución en los ciclos biogeoquímicos, su flujo vertical, y la transferencia de la energía, su conocimiento en el ambiente natural ha tenido un modesto incremento desde su descubrimiento hace más de 100 años (Hofman \& Klinck, 2001). De forma esencial, los estudios de plancton en la región de estudio son exiguos tanto en el fitoplancton (Rojas-Herrera et al., 2012; Meave-del Castillo, Zamudio-Resendiz, \& Castillo-Rivera, 2012; Moreno-Díaz et al., 2015) como en el zooplancton (Balleza-García \& Nestor-Cervantes, 1992; Martínez, 2004; Ramírez-Rosas, 2004).

Los poliquetos pelágicos constituyen un grupo de escasa representación en el zooplancton, en cuanto al número de especies, a 
la abundancia relativa, y a su pequeña talla, por lo que tienen escasa significación en la biomasa total del zooplancton (Orensanz \& Ramírez, 1973); sin embargo su importancia es reconocida en las cadenas tróficas de los océanos del mundo debido a que algunas especies son voraces depredadoras en la comunidad planctónica y, a su vez, son fuente de alimento para varios tipos de peces (Pettibone, 1963; Fernández-Álamo, 1983).

Desde un punto de vista global y comparado con el bentos, son pocos los registros de los anélidos poliquetos que habitan en la comunidad pelágica, tanto de las formas meroplanctónicas (Bhaud, 1966, 1987) como de las holoplantónicas (Dales, 1955, 1957; Fernández-Álamo, 1996). En particular, los trabajos realizados en los mares mexicanos son notoriamente escasos. Para el interior de la Bahía de Acapulco, en el Pacífico, Rioja (1939) describe aspectos de las formas larvarias y postlarvarias de la familia Spionidae y respecto a las comunidades del holoplancton no hay antecedentes, siendo lo más cercano, el estudio de Fernández-Álamo (no publicado) realizado frente a la región nerítica aledaña a la bahía.

Tomando en consideración la exigua información sobre este grupo de importantes gusanos marinos, este trabajo tiene la finalidad de conocer la estructura de esta comunidad y su relación con los periodos de lluvias y secas, así como con las características de la temperatura, la salinidad y la concentración de clorofila- $a$ del agua, en las localidades de muestreo, para contribuir al conocimiento de la comunidad de poliquetos que habita en el plancton de la Bahía de Acapulco, México.

\section{MATERIALES Y MÉTODOS}

Sitio de estudio: En el Pacífico mexicano, la Bahía de Acapulco, del estado de Guerrero (16 $47^{\prime} 54^{\prime \prime}$ y $17^{\circ} 31^{\prime} 55^{\prime \prime} \mathrm{N}-99^{\circ} 50^{\prime} 52^{\prime \prime}$ y $\left.99^{\circ} 56^{\prime} 00^{\prime} \mathrm{W}\right)$, tiene una forma semicircular, con un diámetro aproximado de $6 \mathrm{~km}$ y un área de $\sim 1900 \mathrm{~km}^{2}$; está comunicada con el Océano a través de una boca de alrededor de $3 \mathrm{~km}$ de ancho, que por la presencia de dos penínsulas, junto con la isla de la Roqueta restringen el libre acceso de corrientes, oleaje y viento del exterior (Fig. 1); tiene una profundidad máxima de $\sim 40 \mathrm{~m}$ (Secretaria de Marina [SEMAR], 1977; Nava-Sánchez, 2003; Rosales et al., 2004). La circulación del agua dentro de la bahía es poco conocida, pero SEMAR (1977) describe que en el canal de Boca Chica se detecta una corriente proveniente del oeste que avanza a una velocidad de $\sim 2 \mathrm{~km} / \mathrm{h}$ y junto con los vientos que viajan en la misma dirección producen una corriente paralela a lo largo de la costa de la bahía y sale a la altura de Punta Bruja. Durante septiembre y febrero Meave-del Castillo et al. (2012) detectaron la intrusión de agua de baja temperatura y mayor salinidad lo cual se atribuye a posibles efectos de una surgencia local. De mayo a noviembre la bahía es afectada por depresiones, tormentas y ciclones tropicales (Tamayo, 1970), originando una temporada de lluvias, seguida de una de secas de diciembre a abril. Meave-del Castillo et al. (2012) y Rojas-Herrera et al. (2012) han registrado eutrofización en el primer periodo, atribuida al aumento en los niveles de fosfatos, aportados por el sistema de drenaje de la ciudad de Acapulco, Guerrero.

En total se colectaron 34 muestras de zooplancton en 17 localidades durante mayo y diciembre de 2013, a bordo de una panga con motor fuera de borda. En los arrastres superficiales se utilizó una red de plancton de $31 \mathrm{~cm}$ de apertura de boca, $1.28 \mathrm{~m}$ de manga y $315 \mu \mathrm{m}$ de luz de malla, durante 5 minutos a una velocidad de $\sim 2 \mathrm{~km} / \mathrm{h}$. Las muestras se fijaron con formaldehido al $5 \%$, neutralizado con borato de sodio. Los parámetros ambientales (temperatura, salinidad y clorofila- $a$ ) se midieron con una sonda multiparametros (YSI6600V2-4), previamente calibrada. En el laboratorio todos los poliquetos fueron separados del total de cada muestra, usando un microscopio estereoscópico Zeizz (10-40x) y pinzas de punta fina; posteriormente fueron determinados hasta el nivel taxonómico más bajo, de acuerdo a Rioja (1939, 1941) y Fernández-Álamo (1983); se corroboró la validez de los nombres de los 


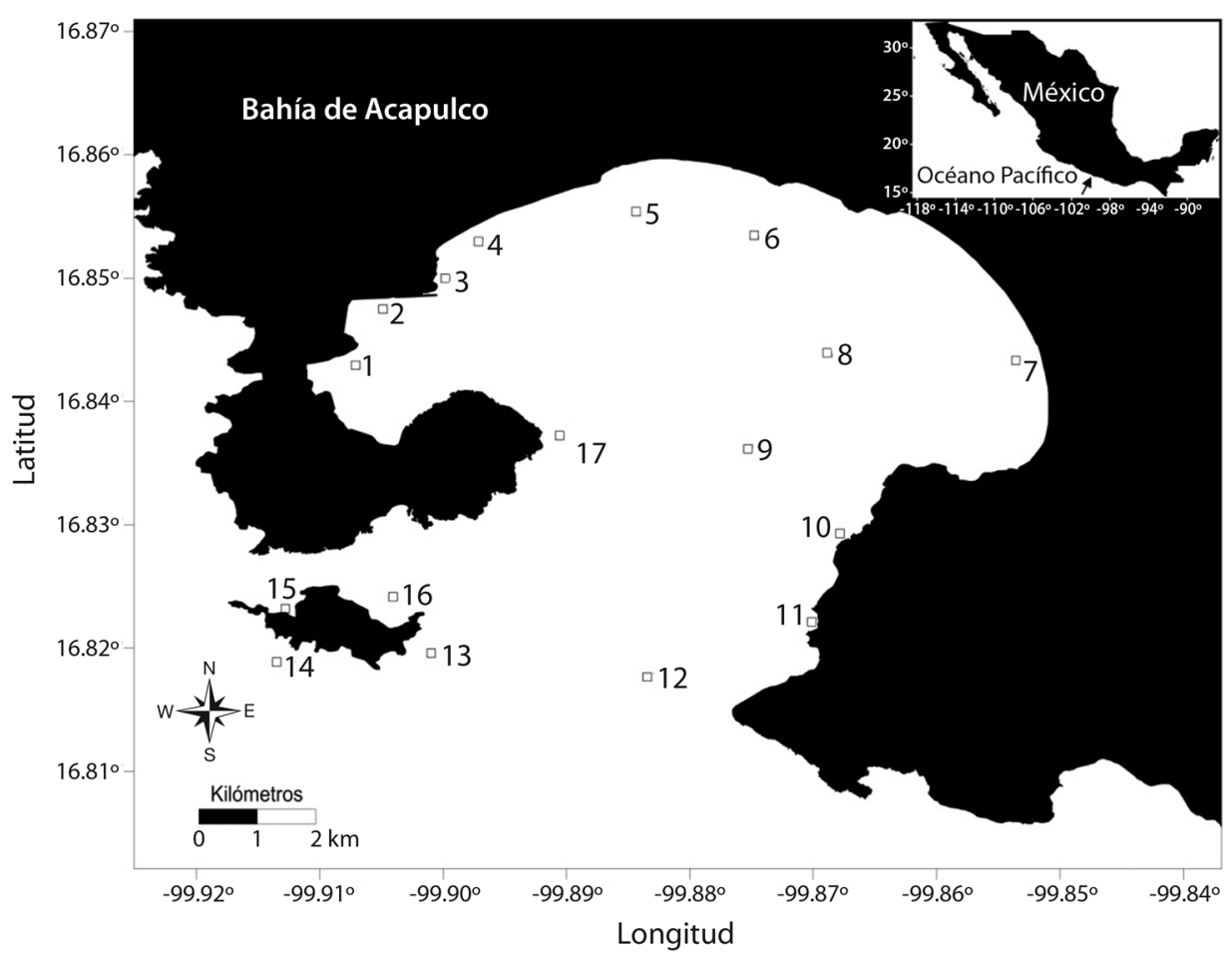

Fig 1. Localización de los sitios de colecta durante mayo y diciembre del 2013, en la Bahia de Acapulco, Guerrrero.

Fig. 1. Location of collection sites during May and December 2013, in the Bahia of Acapulco, Guerrrero.

taxones determinados en World Register of Marine Species [WoRMS] (2018).

Los organismos separados se contaron y se extrapolaron a $100 \mathrm{~m}^{3}$ de agua filtrada (ind $/ 100 \mathrm{~m}^{3}$ ). La caracterización ecológica de los taxones determinados se basó en la prueba de asociación no paramétrica de OlmsteadTukey (Steel \& Torrie, 1985; Sokal \& Rohlf, 1998) y de acuerdo a Varona-Cordero \& Gutiérrez (2003), se definieron en cuatro categorías ecológicas donde: las especies dominantes, son aquellas que presentan la densidad y la frecuencia mayor a la mediana, constantes presentan densidades menores a la mediana y la frecuencia mayor a la mediana, las ocasionales son las que presentan densidad mayor a la mediana y frecuencia menor a la mediana y las raras presentan densidades y frecuencias menores de ambas medianas.

El material estudiado se encuentra depositado en la Colección de Zooplancton del
Laboratorio de Invertebrados de la Facultad de Ciencias, Universidad Nacional Autónoma de México.

\section{RESULTADOS}

Parámetros ambientales: Con relación a los factores ambientales registrados en el agua superficial del área de estudio, en mayo el intervalo de variación en la temperatura fue de $25.4{ }^{\circ} \mathrm{C}$ a $29.3{ }^{\circ} \mathrm{C}$, con un valor promedio de $25.4{ }^{\circ} \mathrm{C}$, mientras que en diciembre los valores oscilaron entre $28.1{ }^{\circ} \mathrm{C}$ y $29.3{ }^{\circ} \mathrm{C}$, con un promedio de $28.9^{\circ} \mathrm{C}$. La salinidad registró una variación de 32.1-33.9 ups, con los valores promedio más altos (33.9 ups) en mayo y los más bajos en diciembre (32.1 ups). La concentración de clorofila- $a$ fue relativamente similar en los dos periodos estudiados, oscilando de 1.9 a $2.4 \mu \mathrm{m} / 1$. La precipitación anual fue de $1270.6 \mathrm{~mm}$, registrándose en mayo $69.6 \mathrm{~mm}$ 
y en diciembre $4.4 \mathrm{~mm}$ (Comisión Nacional del Agua [CONAGUA], 2013).

Estructura de la comunidad: En las dos épocas estudiadas se cuantificaron y determinaron un total de 1873 organismos de los cuales 1685 se presentaron en mayo y 188 en diciembre. La fauna de poliquetos en las muestras de plancton estuvo integrada por 13 familias con un claro predominio de nueve familias meroplanctónicas representadas por las larvas de los poliquetos del bentos, comparada con cuatro holoplanctónicas. Sabellariidae, Spionidae, Chaetopteridae, Magelonidae, Iospilidaey Typhloscolecidae fueron registradas en ambos meses, mientras que Aphroditidae, Syllidae, Phyllodocidae y Alciopidae fueron exclusivas de mayo. Además Serpulidae, Sabellidae y Lopadorhynchidae solo se encontraron en diciembre.
En mayo los sabeláridos presentaron la mayor densidad promedio (423.4 ind $/ 100 \mathrm{~m}^{3}$ ), seguida por Aphroditidae $\left(22.7 \mathrm{ind} / 100 \mathrm{~m}^{3}\right)$ y el menor fue para Phyllodocidae $\left(0.2 \mathrm{ind} / 100 \mathrm{~m}^{3}\right)$; en diciembre el mayor valor fue para Spionidae $\left(17.6 \mathrm{ind} / 100 \mathrm{~m}^{3}\right)$, seguida de Sabellariidae $\left(15.3 \mathrm{ind} / 100 \mathrm{~m}^{3}\right)$ y Magelonidae (13.8 ind $/ 100 \mathrm{~m}^{3}$ ) y el menor para Lopadorhynchidae y Chaetopteridae $\left(0.3 \mathrm{ind} / 100 \mathrm{~m}^{3}\right)$ (Tabla 1$)$.

De las familias de poliquetos holoplanctónicos solo Typhloscolecidae, representada por Typhloscolex muelleri, se registró, en ambos periodos de estudio; en mayo, también se encontraron a Iospilidae con Iospilus phalacroides y Phalacrophorus uniformis y Alciopidae con Alciopina parassitica con dos juveniles. En diciembre, además se observó a Lopadorrhynchidae con un ejemplar de Lopadorrhynchus brevis.

TABLA 1

Composición de las familias de poliquetos del zooplancton en mayo y diciembre del 2013 en la Bahía de Acapulco, Guerrero, México

TABLE 1

Composition of the zooplankton polychaete families in May and December 2013 in Acapulco Bay, Guerrero, Mexico

\begin{tabular}{|c|c|c|c|c|c|c|c|c|c|}
\hline \multirow{2}{*}{ Familia } & \multicolumn{4}{|c|}{ Mayo } & \multicolumn{4}{|c|}{ Diciembre } & \multirow[b]{2}{*}{$\mathrm{H}^{1}$} \\
\hline & $\mathrm{D}^{1}$ & $\mathrm{D}^{2}$ & FA $\%$ & $\mathrm{CE}$ & $\mathrm{D}^{1}$ & $\mathrm{D}^{2}$ & $\mathrm{FA} \%$ & $\mathrm{CE}$ & \\
\hline Alciopidae Ehlers, 1864 & 0.4 & 0.1 & 6 & $\mathrm{R}$ & & & & & $\mathrm{H}$ \\
\hline Aphroditidae Malmgren, 1867 & 22.7 & 4.9 & 53 & $\mathrm{D}$ & & & & & M \\
\hline Chaetopteridae Audouin\&Milne Edwards, 1833 & 4.5 & 1.0 & 29 & $\mathrm{D}$ & 0.3 & 0.6 & 6 & $\mathrm{R}$ & M \\
\hline Iospilidae Bergström, 1914 & 0.8 & 0.2 & 18 & $\mathrm{C}$ & & & & & $\mathrm{H}$ \\
\hline Lopadorrhynchidae Claparede, 1870 & & & & & 0.3 & 0.5 & 6 & $\mathrm{R}$ & $\mathrm{H}$ \\
\hline Magelonidae Cunningham\&Ramage, 1888 & 1.5 & 0.3 & 18 & $\mathrm{D}$ & 13.8 & 25.8 & 29 & $\mathrm{D}$ & M \\
\hline Phyllodocidae Örsted, 1843 & 0.2 & 0.1 & 6 & $\mathrm{R}$ & & & & & M \\
\hline Sabellariidae Johnston, 1865 & 423.4 & 90.5 & 76 & $\mathrm{D}$ & 15.3 & 27.5 & 53 & $\mathrm{D}$ & M \\
\hline Sabellidae Latreille, 1825 & & & & & 3.3 & 5.7 & 47 & $\mathrm{D}$ & M \\
\hline Serpulidae Rafinesque, 1815 & & & & & 0.5 & 1.0 & 12 & $\mathrm{R}$ & M \\
\hline Spionidae Grube, 1850 & 12.5 & 2.7 & 41 & $\mathrm{D}$ & 17.6 & 34.3 & 59 & $\mathrm{D}$ & M \\
\hline Syllidae Grube, 1850 & 0.4 & 0.1 & 6 & $\mathrm{R}$ & & & & & M \\
\hline Typhloscolecidae Uljanin, 1878 & 1.3 & 0.3 & 12 & $\mathrm{O}$ & 0.8 & 1.4 & 12 & $\mathrm{R}$ & $\mathrm{H}$ \\
\hline Total & 467.8 & & & & 51.9 & & & & \\
\hline
\end{tabular}

$\mathrm{D}^{1}=$ densidad promedio (ind $\left./ 100 \mathrm{~m}^{3}\right), \mathrm{D}^{2}=$ densidad relativa $(\%), \mathrm{FA}(\%)=$ frecuencia de aparición, $\mathrm{CE}=$ categoría ecológica (D: dominantes, C: constantes, O: ocasionales, R: raras), $\mathrm{H}^{1}=$ Hábitat (M: meroplanctónica, H: holoplanctónica)

$\mathrm{D}^{1}=$ average density $\left(\mathrm{ind} / 100 \mathrm{~m}^{3}\right), \mathrm{D}^{2}=$ relative density $(\%), \mathrm{FA}(\%)=$ frequency of occurrence, $\mathrm{CE}=$ ecological category (D: dominant, C: constant, O: occasional, R: rare), $\mathrm{H}^{1}=$ Habitat (M: meroplanktonic, $\mathrm{H}$ : holoplanktonic) 
Registro de nuevos taxones: Las larvas de las familias Aphroditidae, Chaetopteridae, Magelonidae, Phyllodocidae, Sabellariidae, Sabellidae, Serpulidae y Syllidae, así como A. parassitica (Alciopidae), I. phalacroides y P. uniformis (Iospilidae), L. brevis (Lopadorrhynchidae) y T. muelleri (Typhloscolecidae), constituyen nuevos registros en el zooplancton de la región de estudio. Los adultos de todas las familias han sido observados en las costas del estado de Guerrero (Rioja, 1939, 1941; Salazar-Vallejo, de León-González, \& Salaices-Polanco, 1989), excepto Magelonidae que constituye posiblemente un nuevo registro en las aguas interiores de la bahía.

Durante mayo, de acuerdo al análisis de Olmstead-Tukey, se registraron cinco familias dentro de la categoría ecológica dominante (Magelonidae, Chaetopteridae, Spionidae, Aphroditidae y Sabellariidae), las cuales aportaron el $99.4 \%$ de la densidad relativa. Otras tres familias se registraron como raras (Alciopidae, Phyllodocidae y Syllidae). Iospilidae se registró como constante, por su alta frecuencia y baja densidad y Typhloscolecidae se consideró ocasional, por su baja frecuencia y alta densidad (Fig. 2A). En diciembre se registraron cuatro familias dentro de la categoría ecológica, dominante (Magelonidae, Spionidae, Sabellidae y Sabellariidae) las cuales contribuyeron con un $93.4 \%$ de la densidad relativa y las otras cuatro familias (Chaetopteridae, Typhloscolecidae, Serpulidae, Lopadorrhynchidae) por su baja abundancia y frecuencia se caracterizaron como raras (Fig. 2B).

\section{DISCUSIÓN}

En general, los parámetros físico-químicos presentaron valores similares a lo registrado previamente (Domínguez-Parra, 1979; GarateLizárraga, Chávez-Almazán, López-Silva, \& Licea, 2012; Meave-del Castilloet al., 2012; Rojas-Herrera et al., 2012; Moreno-Díaz et al., 2015). La aparente incongruencia de los datos de la salinidad con mayores valores en mayo y menores en diciembre, puede explicarse a que los periodos de estudio se ubican justo en la
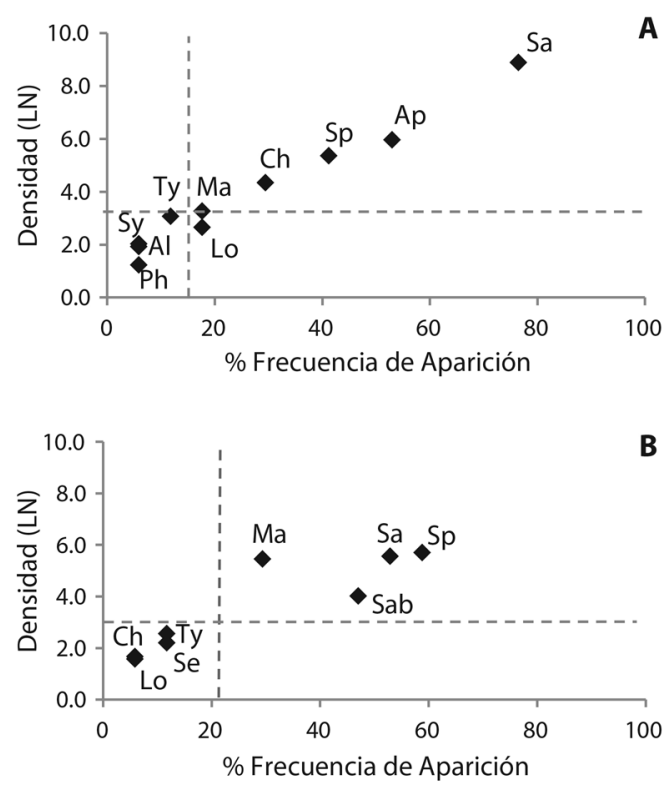

Fig 2. Caracterización ecológica de las familias de poliquetos de Bahía de Acapulco, Guerrero. A) mayo y B) diciembre. (Al) Alciopidae, (Ap) Aphroditidae, (Ch) Chaetopteridae, (Io) Iospillidae, (Lo) Lopadorrhynchidae, (Ma) Magelonidae, (Ph) Phyllodocidae, (Sa) Sabellariidae, (Sab) Sabellidae, (Se) Serpulidae, (Sp) Spionidae, (Sy) Syllidae y (Ty) Typhloscolecidae.

Fig 2. Ecological characterization of families of polychaetes in Acapulco Bay, Guerrero. A) May and B) December. (Al) Alciopidae, (Ap) Aphroditidae, (Ch) Chaetopteridae, (Io) Iospillidae, (Lo) Lopadorrhynchidae, (Ma) Magelonidae, (Ph) Phyllodocidae, (Sa) Sabellariidae, (Sab) Sabellidae, (Se) Serpulidae, (Sy) Syllidae, and (Ty) Typhloscolecidae.

transición de las épocas de lluvias y de secas, de tal forma que el muestreo de mayo se realizó cuando la precipitación de lluvia recién inicia su aparición, mientras que en diciembre dicha temporada ha finalizado, pero aún persiste la influencia de la temporada lluviosa en el agua superficial del mar.

Los resultados en la densidad, la frecuencia de aparición y la riqueza de los poliquetos meroplanctónicos fueron notoriamente contrastantes en las temporadas estudiadas; el total de larvas capturadas $\left(7952.0 \mathrm{ind} / 100 \mathrm{~m}^{3}\right)$ en mayo, coincidió, con el promedio de temperatura más bajo $\left(25.4{ }^{\circ} \mathrm{C}\right)$, y el promedio de salinidad más alto (33.9 ups), siendo importante considerar que dicha diferencia está definida por la alta 
densidad relativa $(90.5 \%)$ de las larvas de la familia Sabellariidae, lo que indica que en este mes se presentó un máximo valor que podría corresponder una alta intensidad de la etapa reproductiva. Al analizar la distribución y la abundancia de estas larvas se registraron los valores más altos justo en la boca de la bahía lo que puede estar indicando que la distribución de los adultos se encuentra fuera y que las larvas se encuentran entrando a una zona menos expuesta para su mejor desarrollo o bien que la colonia o colonias de sabeláridos se ubican dentro de la bahía, al respecto, Rioja (1942) registró masas de tubos de arena de estos gusanos en la zonas intermareal y submareal y posiblemente las larvas tratan de salir hacia la región nerítica.

La menor densidad (882.1 ind $\left./ 100 \mathrm{~m}^{3}\right)$ y riqueza de las familias de poliquetos en el plancton se registró en diciembre, en coincidencia con valores altos de temperatura $\left(29.3^{\circ} \mathrm{C}\right)$ y bajos valores de salinidad (32.1 ups) y de clorofila- $a$. La mayor densidad relativa se observó en Spionidae (34.3\%), Sabelariidae (27.5\%) y Magelonidae (25.8 \%).

Los miembros de la familia Sabellariidae son constructores de arrecifes de arena y por lo tanto tienen un papel primordial en las características estructurales del fondo, conformando grandes masas de tubos adheridos a sustratos duros en la región intermareal, contribuyendo de forma notable en la dinámica del oleaje, aminorando su fuerza y formando ambientes complejos que son utilizados por otros organismos como turbelarios, crustáceos, otros poliquetos, nemertinos cefalópodos, equinodermos y una amplia diversidad de algas (Rioja, 1942; Dollfus, 1960; Dubois, Comtet, Retière, \& Thiébaut, 2007; Ataide, Venekey, Filho, \& dos Santos, 2014; Aviz, Pinto, Ferreira, Rocha, \& Fihlo, 2016). Dentro de la Bahía de Acapulco, los adultos de esta familia fueron registrados por Rioja $(1941,1942,1962)$ indicando que Idanthyrsus pennatus se encuentra formando grandes masas de tubos de arena sobre los cuales se observa una rica e interesante biocenosis (Rioja, 1941, 1942). La presencia de estas larvas en estado de dos o cuatro segmentos que de acuerdo a Eckelbarger (1976) representan un desarrollo de 40 a 46 h y 6 días, respectivamente, permiten asumir que el desove se realizó tanto en mayo como en diciembre, siendo en el primer caso muy abundante. Los periodos registrados en la Bahía de Acapulco coinciden con lo observado por Dubois et al. (2007) en las costas de la Bahía del Monte Saint Michel en Francia, que aparecen en los mismos periodos de este estudio. Los adultos de las otras familias bentónicas registradas como larvas en este estudio, ya han sido mencionadas de forma general en el estado de Guerrero (SalazarVallejo et al., 1989), excepto Magelonidae que no presenta ningún registro en esta región del Pacífico mexicano, sin embargo se requiere de una revisión más detallada para incluirla como nuevo registro para toda la zona costera de esta región.

El predominio de las familias meroplanctónicas con respecto a las holoplanctónicas en el interior de la bahía de Acapulco, coincide con la distribución biogeográfica de estos gusanos, debido a que se distribuyen principalmente en las regiones oceánicas y su presencia en las áreas costeras es esporádica u ocasional (StøpBowitz, 1948; Chapman \& Dales, 1954; Day, 1967, 1975; Tovar, 2012). Particularmente, es interesante destacar que los ejemplares de A. parassitica fueron formas jóvenes en estado de siete y 15 segmentos, ya que como su nombre lo indica su primer registro fue dentro del ctenóforo Cydippe densa Forskál (Claparède \& Panceri, 1867), por lo que se requiere de un análisis más preciso sobre la biología de esta especie.

Los resultados de este estudio ponen de manifiesto la necesidad de intensificar los muestreos de tipo cualitativo y cuantitativo del zooplancton, para incrementar el conocimiento sobre estos temas, permitiendo establecer desde una perspectiva funcional sus relaciones en la dinámica oceánica tanto del plancton como del bentos.

Este primer estudio en Bahía de Acapulco, proporciona valiosa información de la biodiversidad del zooplancton. Los nuevos registros de las larvas de las familias bénticas y las 
especies de las holoplanctónicas representan un aporte al conocimiento de la comunidad de poliquetos; también cabe mencionar que la notable presencia de las larvas de la familia Sabellariidae, posiblemente indica una intensidad en el proceso reproductivo de estos interesantes poliquetos que son formadores de importantes arrecifes de arena.

Declaración de ética: los autores declaran que todos están de acuerdo con esta publicación y que han hecho aportes que justifican su autoría; que no hay conflicto de interés de ningún tipo; y que han cumplido con todos los requisitos y procedimientos éticos y legales pertinentes. Todas las fuentes de financiamiento se detallan plena y claramente en la sección de agradecimientos. El respectivo documento legal firmado se encuentra en los archivos de la revista.

\section{AGRADECIMIENTOS}

Un reconocimiento especial al Programa de Verano de Investigación Científica de la Academia Mexicana de Ciencias (AMC) por el apoyo brindado a Neysy Gálvez Zeferino para la realización de la estancia de investigación en el verano del 2013 del 24 de junio al 23 de agosto en el Laboratorio de Invertebrados de la Facultad de Ciencias de la Universidad Nacional Autónoma de México. También agradecemos las sugerencias y observaciones de los revisores anónimos que mejoraron la versión final de este trabajo.

\section{RESUMEN}

Introducción: Desde un punto de vista global, los poliquetos que habitan en la comunidad pelágica han sido poco estudiados, aunque son un eslabón importante en las cadenas alimentarias de los ecosistemas marinos. Objetivo: Este trabajo contribuye al conocimiento de esta importante comunidad, analizando la riqueza y la densidad de los poliquetos. Métodos: Las muestras de plancton se tomaron en 17 localidades durante mayo y diciembre de 2013 en el área de estudio, mediante arrastres superficiales en una embarcación con un motor fuera de borda. Utilizamos una red con un diámetro de boca de $0.3 \mathrm{~m}$, una longitud de
$1.30 \mathrm{~m}$ y una abertura de malla de $0.3 \mathrm{~mm}$. De cada muestra recolectada, los poliquetos se separaron y determinaron al nivel taxonómico más bajo posible. Resultados: Cuantificamos un total de 1873 individuos, con una variación importante en la densidad relativa, ya que en mayo se registraron $7952.0 \mathrm{ind} / 100 \mathrm{~m}^{3}$, mientras que en diciembre solo hubo $882.1 \mathrm{ind} / 100 \mathrm{~m}^{3}$. Se analizaron las diferencias en la composición y densidad de los poliquetos determinados, de los cuales la proporción más alta pertenece a las formas larvales de las poblaciones bentónicas (meroplancton) y la más baja a los organismos del holoplancton. Durante los dos periodos de estudio se observaron trece familias, de las cuales, Sabellariidae, Spionidae, Chaetopteridae, Magelonidae, Iospilidae y Typhloscolecidae fueron comunes en ambos periodos, mientras que Aphroditidae, Syllidae, Phyllodocidae y Alciopidae fueron exclusivas de mayo y Serpullidae, Sabellidae y Lopadorhynchidae solo se encontraron en diciembre. Conclusiones: Es importante señalar que las variaciones observadas proporcionan valiosa información sobre los ciclos de vida y los aspectos biogeográficos de los poliquetos en el área de estudio.

Palabras clave: gusanos marinos, zooplancton, ecología, Pacífico mexicano.

\section{REFERENCIAS}

Ataide, M. B., Venekey, V, Filho, J. S. R., \& dos Santos P. J. P. (2014). Sandy reefs of Sabellaria wilsoni (Sabellariidae: Polychaeta) as ecosystem engineers for meiofauna in the Amazon coastal region, Brazil. Marine Biodiversity, 44, 403-413.

Aviz, D., Pinto, A. J. A., Ferreira, M. A. P., Rocha, R. M., \& Fihlo, R. J. S. (2016). Reproductive biology of Sabellaria wilsoni (Sabellariidae: Polychaeta), an important ecosystem engineer on the Amazon coast. Journal of the Marine Biological Association of the United Kingdom, 98(4): 743-754.

Balleza-García, M., \& Néstor-Cervantes F. J. (1992). Contribución al estudio de los quetognatos de la Bahía de Acapulco, Guerrero. (Tesis de licenciatura). Acapulco, Guerrero, México: Universidad Autónoma de Guerrero, Escuela Superior de Ecología Marina.

Bhaud, M. (1966). Les larves planctoniques d'Annélides Polychaétes, leur intéret pour une meilleu reconnaissance faunistique. Vie Milieu, 17(1B), 435-437.

Bhaud, M. (1987). Description and identification of Polychaete larvae; their implications in current biological problems. Oceanis série de documents oceanographiques, 13(Fasc. 6), 597-753

Chapman, G., \& Dales, P. (1954). Aspects of the fauna and flora of Azores. II. Polychaeta. Annals and Magazine of Natural History, 7, 678-683. 
Claparède, E., \& Panceri, P. (1867). Sopra un Alciopide parassito della Cydippe densa Forskál. Att Societa Italiana Di Scienze Naturale, 10, 257-258.

Comisión Nacional del Agua. (2013). Comisión Nacional del Agua, Precipitación a nivel nacional y por identidad federativa. Gobierno de México. Recuperado de https://smn.cna.gob.mx/es/

Dales, R. P. (1955). The pelagic polychaetes of Monterey Bay, California. Annals and Magazine of Natural History Serie, 12(8), 434-444.

Dales, R. P. (1957). Pelagic polychaetes of the Pacific Ocean. Bulletin of the Scripps Institution of Oceanography of the University of California, 7, 99-168.

Day, J. H. (1967). A monograph on the Polychaeta of southern Africa. Part 2. Sedentaria. London, UK: Trustees of the British Museum (Natural History).

Day, J. H. (1975). Zooplancton de la région de Nosy-Bé. 10. The biology of planktonic Polychaeta near NosyBé, Madagascar. Cahiers de l'ORSTOM Série Océanographique, 3, 197-216.

Dollfus, R. (1960). Sur un récifactuel le bang des Hermelles de la baie du Mont-Sant-Michel. Quelques renseignements documentaires. Bulletin de la Societé Geologique de France, 7(2), 133-140.

Domínguez-Parra, S. (1979). Estudio de la calidad reglamentaria y ecológica de las aguas costeras en la Bahía de Acapulco, Guerrero y proximidades realizado de noviembre de 1978 a marzo de 1979. (Tesis de Maestría). Ciudad de México, México: Universidad Nacional Autónoma de México.

Dubois, S., Comtet, T., Retière, C., \& Thiébaut, E. (2007). Distribution and retention of Sabellaria alveolata larvae (Polychaeta: Sabellariidae) in the Bay of Mont-Saint-Michel, France. Marine Ecology Progress Series, 346, 243-254.

Eckelbarger, K. J. (1976). Larval development and population aspects of the reef-building polychaete Phragmatopoma lapidosa from the east coast of Florida. Bulletin of Marine Science, 26(2), 117-132.

Fernández-Álamo, M. A. (1983). Los poliquetos pelágicos (Annelida-Polychaeta) del Pacífico Tropical Oriental: Sistemática y Zoogeografia. (Tesis Doctoral). Ciudad de México, México: Universidad Nacional Autónoma de México.

Fernández-Álamo, M. A. (1996). Holoplanktonic Polychaetes off the Southwestern Coast of Baja California, Mexico, in March, 1977. Anales Instituto de Biología Universidad Nacional Autónoma de México, Serie Zoología, 67 (1), 51-66.

Gárate-Lizárraga, I., Chávez-Almazán, L. A., López-Silva, S., \& Licea, S. (2012). Toxicity and paralytic toxin profile in Pyrodinium bahamense var compressum and violet oyster in Bahía de Acapulco, Guerrero, Mexico. Harmful Algae News, 45, 1-3.

Hofman, E., \& Klinck, J. M. (2001). Marine Zooplankton Colloquium 2. Future marine zooplankton research a perspective. Marine Ecology Progress Series, 222, 297-308.

Martínez, O. S. Y. (2004). Estudio de la productividad de la población de los copépodos pelágicos de la Bahía de Acapulco Guerrero, México. (Tesis de Licenciatura). Acapulco, Guerrero, México: Universidad Autónoma de Guerrero.

Meave-del Castillo, M. E., Zamudio-Resendiz, M. E., \& Castillo-Rivera, M. (2012). Riqueza fitoplatónica de la Bahía de Acapulco y zona costera aledaña, Guerrero, México. Acta Botánica Mexicana, 100, 405-487.

Moreno-Díaz, G., Rojas-Herrera, A. A., Violante-González, J., González-González, J., Rosas Acevedo, J. L., \& García Ibañez, S. (2015). Temporal Variation in Composition and Abundance of Phytoplankton Species during 2011 and 2012 in Acapulco Bay, Mexico. Open Journal of Marine Science, 5, 358-367.

Nava-Sánchez, E. (2003). Riesgo geológico en la zona costera de la bahía de Acapulco, Guerrero (Informe Técnico). Guerrero, México: COREMI.

Orensanz, J. M., \& Ramírez, F. C. (1973). Taxonomía y distribución de los poliquetos pelágicos del Atlántico Sudoccidental. Boletín del Instituto de Biología Marina, 21, 1-86.

Pettibone, M. H. (1963). Marine Polychaete worms of the New England Region. 1. Families Aphroditidae trought Trochochaetidae. Bulletin of United States Natural Museum, 227, 1-356.

Ramírez-Rosas, S. (2004). Evaluación de la diversidad y abundancia de los copépodos pelágicos en la Bahía de Acapulco Guerrero, México. (Tesis de licenciatura). Acapulco, Guerrero, México: Universidad Autónoma de Guerrero.

Rioja, E. (1939). Estudios Anelidológicos I. Observaciones acerca de varias formas larvarias y postlarvarias pelágicas de Spionidae procedentes de Acapulco. Anales del Instituto de Biología, Universidad Nacional Autónoma de México, 10, 297-311.

Rioja, E. (1941). Estudios Anelidológicos III. Datos para el conocimiento de la fauna de poliquetos de las costas del Pacífico de México. Anales del Instituto de Biología, Universidad Nacional Autónoma de México, 12, 297-311.

Rioja, E. (1942). Estudios Anelidológicos VI. Observaciones sobre algunas especies de Sabellaridos de las costas mexicanas del Pacífico. Anales del Instituto de Biología, Universidad Nacional Autónoma de México, 13, 1-155. 
Rioja, E. (1962). Estudios Anelidológicos XXVI. Algunos anélidos poliquetos de las costas del Pacífico de México. Anales del Instituto de Biología, Universidad Nacional Autónoma de México, 33, 131-229.

Rojas-Herrera, A. A., Violante-González, J., García-Ibáñez, S., Sevilla-Torres, V. M. G., Gil-Guerrero, J. S., \& Flores-Rodríguez, P. (2012). Temporal variation in the phytoplankton community of Acapulco Bay, Mexico. Microbiology Research, 3(4), 13-19.

Rosales, J., Uribe, J., Cuevas, S., Ramírez, M., Rivera, J., Arceo y Cabrilla, F., ... Martínez, G. (2004). Atlas de Peligros Naturales de la Ciudad Acapulco, Gro, Identificación y Zonificación (Texto y Anexo Cartográfico). México: SEDESOL COREMI.

Salazar-Vallejo, S. I., de León-González, J. A., \& SalaicesPolanco, H. (1989). Poliquetos (Annelida: Polychaeta) de México: Generalidades, claves ilustradas para familias y géneros y bibliografía lista de especies. La Paz, México: Universidad Autónoma de Baja California Sur.

Secretaria de Marina. (1977). Estudio Geográfico de la región de Acapulco, Guerrero. México. México: Dirección General de Oceanografía.

Sokal, R. R., \& Rohlf, F.J. (1998). Biometry (2nd ed.), San Francisco, USA: W. H. Freeman and Company.
Steel, G. D. R., \& Torrie, H. J. (1985). Bioestadistica: principios y procedimientos (2da ed.). Bogotá, Colombia: McGraw-Hill.

Støp-Bowitz, C. (1948). Polychaeta from the "Michael Sars" North Atlantic deep-sea expedition 1910. Report on the Scientific Results of the Michael Sars North Atlantic Deep-Sea Expedition, 5(8), 1-91.

Tamayo, J. L. (1970). Geografía Moderna de México. México: Editorial Trillas, S.A.

Tovar, F. B. (2012). Taxonomia e Padrões de Distribuição dos Poliquetas Pelágicos (Annelida-Polychaeta) da Região Central da Zona Econômica Exclusiva Brasileira, entre $13^{\circ}$ e $25^{\circ} \mathrm{S}$ e $28^{\circ}$ e $42^{\circ} \mathrm{W}$. (Tesis doctoral). Rio Janeiro, Brazil: Universidad e Federal Fluminense.

Varona-Cordero, F., \& Gutiérrez, M. F. (2003). Estudio multivariado de la fluctuación espacio-temporal de la comunidad fitoplanctónica en dos lagunas costeras del estado de Chiapas. Hidrobiológica, 13(3), 177-194.

World Register of Marine Species (2018). World Register of Marine Species. Recuperado de http://www. marinespecies.org at VLIZ. Accedido 2018-08-29. doi: $10.14284 / 170$. 